\title{
Scour protection design in highly morphodynamic environments
}

\author{
H.J. Riezebos \& T.C. Raaijmakers \\ Deltares, Delft, The Netherlands
}

\author{
A. Tönnies-Lohmann \& S. Waßmuth \\ wpd offshore solutions GmbH, Bremen, Germany
}

P.W. Van Steijn

Deltares, Delft, The Netherlands

ABSTRACT: Offshore wind parks are often developed in areas with a highly morphodynamic seabed. In or-
der to keep the pile fixation level within acceptable limits for pile design, sophisticated scour mitigation strat-
egies are required. This paper discusses the scour protection strategy for the Nordergründe Offshore Wind
Farm, where $10 \mathrm{~m}$ seabed level drops are predicted at some monopile locations. Physical model tests were per-
formed to validate the scour protection design, focussing on correct falling apron behaviour and the stability
of the loose rock scour protection. The outcome of the tests showed good correlation with a modified relative
mobility parameter. Using this parameter, the scour protection was optimised for the individual pile locations.

\section{INTRODUCTION}

Offshore wind farms are often developed in areas with a highly morphodynamic seabed. The morphodynamic activity can be caused by migrating sand waves, tidal flats or channels. Sophisticated scour mitigation strategies are required in order to keep the pile fixation level within acceptable limits for pile design. This paper discusses the scour protection strategy for the offshore wind turbines of the Nordergründe Offshore Wind Farm (NOWF), which will be installed on monopile foundations in the Jade-Weser-Estuary, offshore Germany.

The bathymetry of NOWF is highly morphodynamic. Earlier studies by Deltares addressed the morphology in the area and found potential maximum seabed level variations in the order of ten metres for some foundations. Relatively abrupt seabed level lowering can occur due to the migration of a flood channel adjacent to the park's outline, more gradual lowering can be caused by erosion of the sandbank on which the wind farm is located. The foundations of the wind turbine generators of NOWF should be able to cope with the predicted potential seabed level variations.

\subsection{Scour mitigation strategies}

Three mitigation strategies (illustrated in Fig. 1) can be considered to deal with large scale seabed level variations:

1. Adjust the monopile design to withstand all potential seabed level variations;
2. Immediately protect the initial seabed, preventing seabed level variations at the pile base;

3. Monitor the bathymetry and install scour protection at a later phase.

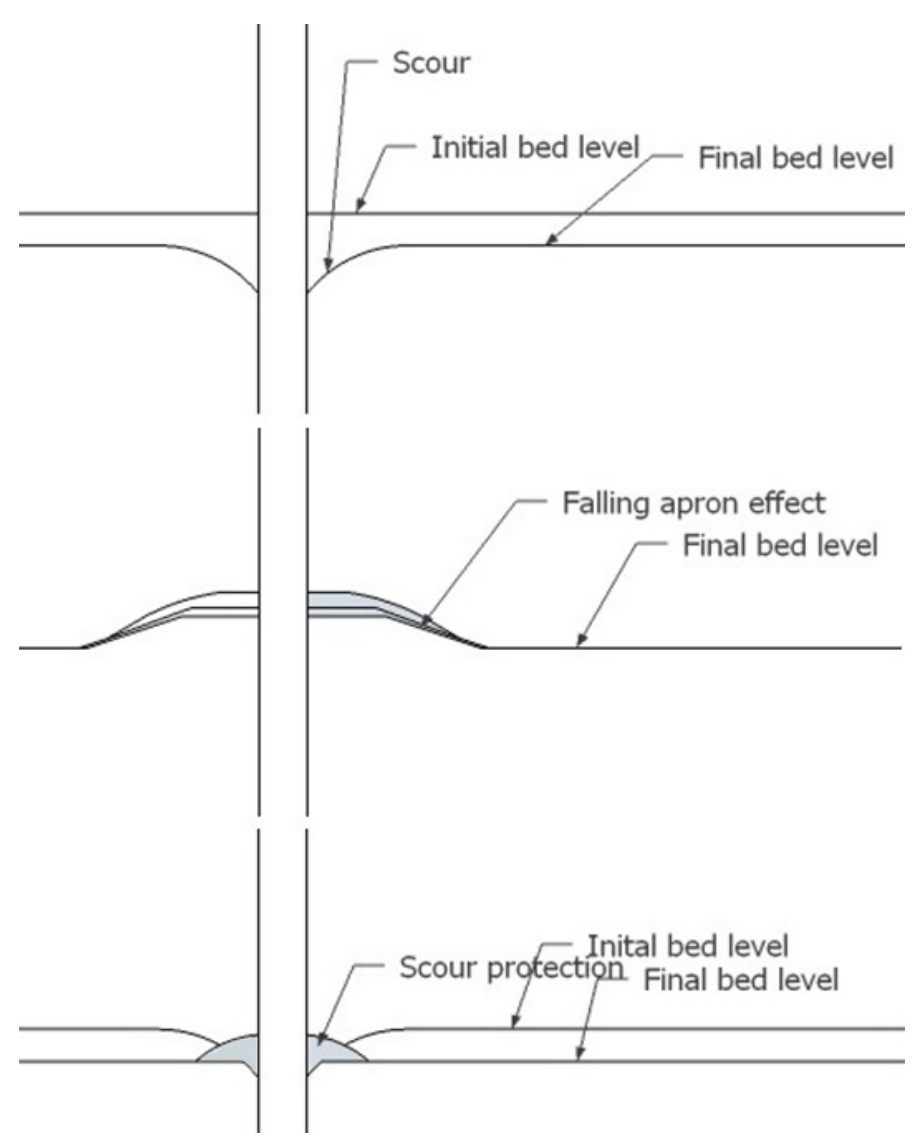

Figure 1. Scour mitigation strategies illustrated for a lowering seabed: 1) no protection with free scour development (top), 2) immediate protection (middle), 3) monitor and react (bottom). 
Due to the very large predicted variations in seabed level for NOWF both Strategy 1 and Strategy 2 are considered to be not economic. In Strategy 1 the pile design should be adapted so that the foundation can withstand the extreme seabed level variations. However, for NOWF the potential range in fixation depth is so extreme that a functioning pile design (e.g. with respect to the resonance frequency) is virtually impossible. Strategy 2 (fixation of the initial seabed level by means of a scour protection) ensures a constant fixation depth of the pile, allowing for optimisation of the pile design. For this strategy, however, the design of the scour protection becomes problematic. Due to the predicted seabed level lowering the protection would be located relatively high in the water column, resulting in very severe hydrodynamic loading. To be able to withstand the design load conditions, very large rock would be required as armour for the scour protection. Such large diameter armour rock would then require at least one (and probably more) filter layers. At the same time this multi-layered scour protection would have to be able to resist the predicted seabed level lowering. The predicted seabed level lowering will have to be controlled by means of a falling apron: material rolls down at the edge of the scour protection, stabilising the slope and preventing further lowering. In this way the seabed lowering is kept at a sufficiently large distance from the base of the foundation. A multi-layered falling apron (i.e. an armour layer and one or more filter layers) is however unfeasible, because the proper layering of the material throughout the falling apron process (which is essential for a correctly functioning filter) cannot be guaranteed.

Strategy 3, allowing a scour hole to develop and installing the scour protection once the scour reaches a certain (pre-determined) depth, was selected as the most viable option. In this strategy the pile experiences a comparatively small variation in fixation depth, while the required rock volume is reduced. First a conceptual scour protection layout for NOWF was developed, according to this strategy. This layout was subsequently tested and validated by means of physical model tests in Deltares' Atlantic Basin.

\section{SCOUR PROTECTION CONCEPT}

As explained in the introduction, the scour protection should be flexible enough to handle a seabed level lowering of up to 10 metres. It also has to withstand the design storm conditions, both in the initial situation and in the potential situation when largescale morphologic change has occurred. The dimensions of the conceptual scour protection design were determined based on these boundary conditions.

\subsection{Rock grading}

It was decided to use a single-graded scour protection, with 3-9" rock material $\left(\mathrm{D}_{50}=0.15 \mathrm{~m}\right)$. According to Strategy 3, the material will be partly installed inside a pre-developed scour hole. To our knowledge there is no theory available to accurately take into account the effect of the material's sheltered position inside the scour hole. Initial mobility calculations therefore ignored the sheltering effect, thus arriving at a conservative estimate of the rock mobility. The calculations showed that in the initial situation (without large-scale morphological change) the scour protection would be dynamically stable. Some reshaping of the scour protection is expected to occur, but well within acceptable limits.

\subsection{Layer thickness}

The required thickness of the single-graded scour protection is mainly determined by the filter criterion. The 3-9" rock grading is a geometrically open filter, meaning that sediment can escape through the pores of the grading. Loss of sediment through the scour protection (the winnowing effect) should however be prevented. Typically this is achieved by ensuring a sufficient layer thickness of the protection.

An overview of the applicable theory on the application of open filters at circular piers is given in De Sonneville (2014). There it was found that existing formulations on the design of open filters either do not take into account the presence of a pier/monopile or do not consider the combined effect of waves and currents. None of the existing formulations are therefore applicable for open filters at monopiles in typical offshore conditions. In this paper the theory by Hoffmans (2012) is used, in which a relation for geometrically open filters in non-uniform flow is derived. This relation is visualised in Figure 2. Given the conditions in NOWF, this would result in a minimum layer thickness of approximately $1 \mathrm{~m}$.

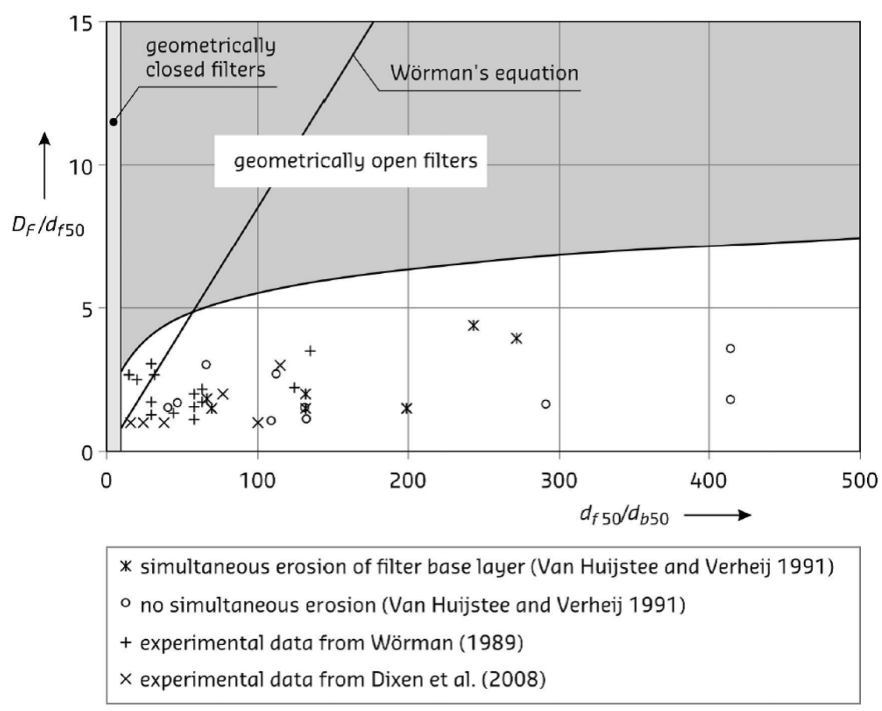

Figure 2. Logarithmic relation for required filter layer thickness according to Hoffmans (2012). 
The pre-developed scour hole will be filled with rock till a level of $-2 \mathrm{~m}$ relative to the original seabed. Based on the predicted scour depth this method ensures a layer thickness of several metres at the pile face. Further from the pile the thickness decreases, till a minimum thickness of $1 \mathrm{~m}$. This concept is illustrated in Figure 3.

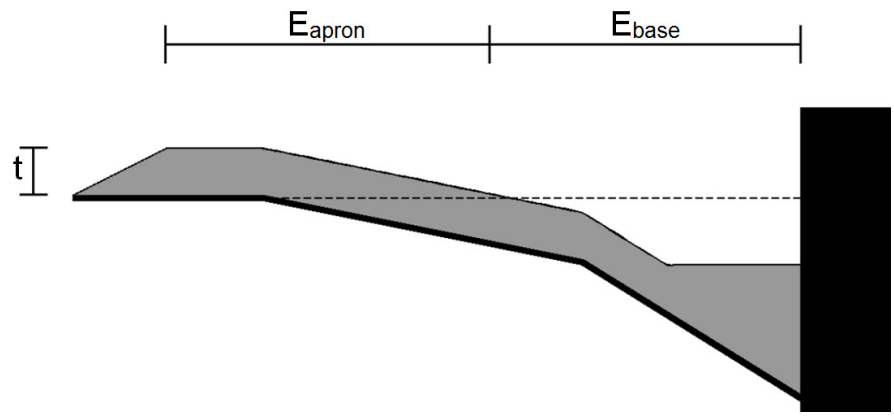

Figure 3. Cross-section of the scour protection concept and main dimensions.

\subsection{Extent}

The required extent of the scour protection is formed by two components: a base extent $\left(\mathrm{E}_{\text {base }}\right)$ and an additional falling apron extent $\left(\mathrm{E}_{\text {apron }}\right)$. This is illustrated in Figure 3. The base extent forms the minimum required extent of the scour protection. The additional falling apron extent is required for dealing with the predicted large-scale seabed level drops. The base extent is based on the minimum required scour protection diameter of four pile diameters $\left(4^{*} D_{\text {pile }}\right)$, which is a commonly accepted value for the extent of a monopile scour protection. Based on this, the extent from the pile face is $1.5^{*} \mathrm{D}_{\text {pile. }}$.

For the determination of the falling-apron extent, use was made of the formula by Van Velzen (2012):

$r_{f, 0}=\sqrt{\frac{\frac{\pi}{6}\left(L_{2}^{3}-r_{f, A}^{3}\right)-\frac{\pi}{3} \tan y\left(\left(L_{2}-d_{f, 50}\right)^{3}-\left(L_{2}-d_{f, 50}-\frac{h_{b d}}{\tan y}\right)^{3}\right)}{\pi D_{f, 0}}}$

With:

$$
\begin{aligned}
& L_{2}=r_{f, A}+2\left(D_{f, 0}+h_{b d}\right) ; \\
& \tan \gamma=\tan \left(a \tan \left(\frac{1}{2}\right)-a \tan \left(\frac{D_{f, A}-d_{f, 50}}{\sqrt{5}\left(D_{f, 0}+h_{b d}\right)-\frac{2 \cdot d_{f, 50}}{\sqrt{5}}}\right)\right) ;
\end{aligned}
$$

pile diameter, $\mathrm{D}_{\text {pile }}$; total extent, $\mathrm{r}_{\mathrm{f}, 0}$; base extent, $\mathrm{r}_{\mathrm{f}, \mathrm{A}}$; thickness, $\mathrm{D}_{\mathrm{f}, 0}$; falling apron thickness, $\mathrm{D}_{\mathrm{f}, \mathrm{A}}$ (assumed to be equal to $\mathrm{D}_{\mathrm{f}, 0}$ ); bed degradation $\mathrm{h}_{\mathrm{bd}}$ and thickness at falling apron base, $\mathrm{d}_{\mathrm{f}, 50}$ (assumed to be $0.2 \mathrm{~m}$ ). The formula provides the required extent for a single-graded scour protection, based on the base extent, the rock grading and the predicted seabed level drop. An overview of the corresponding geometry is provided in Figure 4.

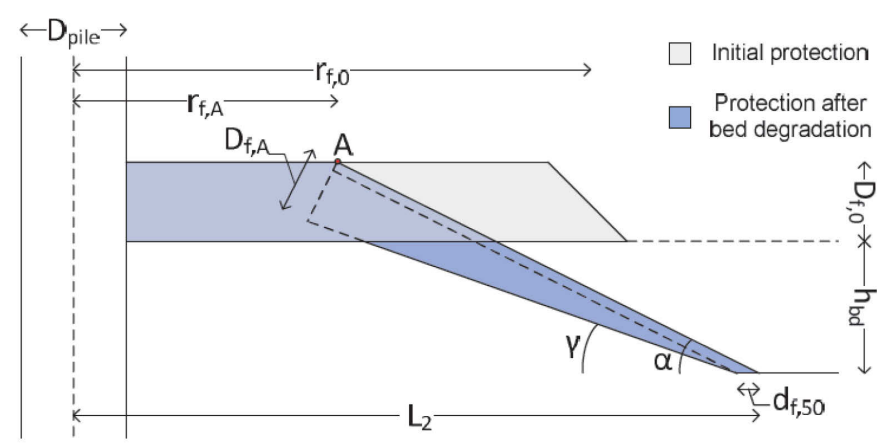

Figure 4. Cross-sections of the initial protection and the falling apron geometry (Van Velzen, 2012).

The formula by Van Velzen was derived for statically stable scour protections in current-only conditions. Although it is not necessarily applicable for the NOWF case (where a dynamically stable scour protection is exposed to combined current and waves), this formula was taken as a starting point in this research to determine the required falling apron volume. Based on the model tests the falling apron volumes were adjusted (see next section).

\subsection{Additional layouts in the physical model tests}

In combined current and waves more rock volume may be required than the volume based on the formula by Van Velzen for current-only conditions. Therefore, the following three scour protection layouts were considered in the test programme:

1. Layer thickness (t) of $1 \mathrm{~m}$ and apron extent ( $\left.E_{\text {apron }}\right)$ according to Van Velzen, 2012.

2. Layer thickness $(\mathrm{t})$ of $1.2 \mathrm{~m}$ and apron extent increased by $20 \%$ (i.e. $E_{\text {apron }}=1.2 \cdot\left(\mathrm{r}_{\mathrm{f}, 0}-\mathrm{r}_{\mathrm{f}, \mathrm{A}}\right)$ )

3. Layer thickness (t) of $1.5 \mathrm{~m}$ and apron extent increased by $44 \%$ (i.e. $\mathrm{E}_{\text {apron }}=1.44 \cdot\left(\mathrm{r}_{\mathrm{f}, 0}-\mathrm{r}_{\mathrm{f}, \mathrm{A}}\right)$ ).

Furthermore, to improve the stability of the protection also the use of high density material (HD) is investigated in the model tests (with a particle density of $3000 \mathrm{~kg} / \mathrm{m}^{3}$ ), which was compared to the stability of normal density material (ND) with $2650 \mathrm{~kg} / \mathrm{m}^{3}$. To have similar shaped material in the model as in the field, the rock used in the model tests was obtained from the same quarries which also supplied the rock at prototype scale.

\section{OVERVIEW OF PHYSICAL MODEL TESTS}

The viability of the conceptual scour protection layouts introduced in Section 2, was investigated in a physical model test programme.

\subsection{Test facility}

All tests were performed in Deltares' Atlantic Basin. This basin has a length of $75 \mathrm{~m}$ and a width of $8.7 \mathrm{~m}$. The sandy test section in the middle of the basin is $15 \mathrm{~m}$ long and spans the width of the basin. The ba- 
sin has three pumps, with a total discharge capacity of $3 \mathrm{~m}^{3} / \mathrm{s}$. The basin's wave generator can create waves with a significant wave height up to $0.25 \mathrm{~m}$, in water depths up to $1 \mathrm{~m}$.

The properties of the Atlantic Basin allow for accurate reproduction of offshore storm conditions; high waves can be combined with strong currents. Also tidal conditions can be simulated accurately, because the pumps can discharge in both directions. This is vital for investigating the falling apron effect, as a unidirectional current would result in an asymmetric launching of the falling apron.

\subsection{Test setup and measurement techniques}

An overview of the test setup is provided in Figure 5. Two identical models of a monopile are placed side by side in the basin's test section. This allows for testing two different scour protection layouts simultaneously, under identical conditions. The models consist of a Perspex tube (diameter $20 \mathrm{~cm}$ ), which is connected to the concrete floor beneath the $45 \mathrm{~cm}$ thick sand bed $\left(\mathrm{d}_{50 \text {; sand }}=180 \mu \mathrm{m}\right)$. The models are equipped with an internal camera system with fish-eye lens, which enables the measurement of deformation along the pile face. This technique was developed in-house at Deltares in 2006 and was further developed since then; see e.g. Raaijmakers et al. (2008) and De Sonneville (2010). Bathymetry measurements are taken before and after each test by means of stereophotography. With this technique a $3 \mathrm{D}$ bathymetry is obtained, as well as a 3D colour image. Measurements can be taken both when the basin is dry, and when a refraction surface is present (e.g. when there is water in the basin). The stereophotography technique (both dry and underwater) is extensively discussed in Raaijmakers et al. (2012).

During the tests the progression of the falling apron effect is observed by means of underwater cameras. Two cameras are installed at each model, one aimed upstream and one downstream of the scour protection. Measurement of the hydrodynamic conditions is done with an array of electromagnetic flow meters (type EMF-30, developed in-house at Deltares) and resistance-type wave gauges.

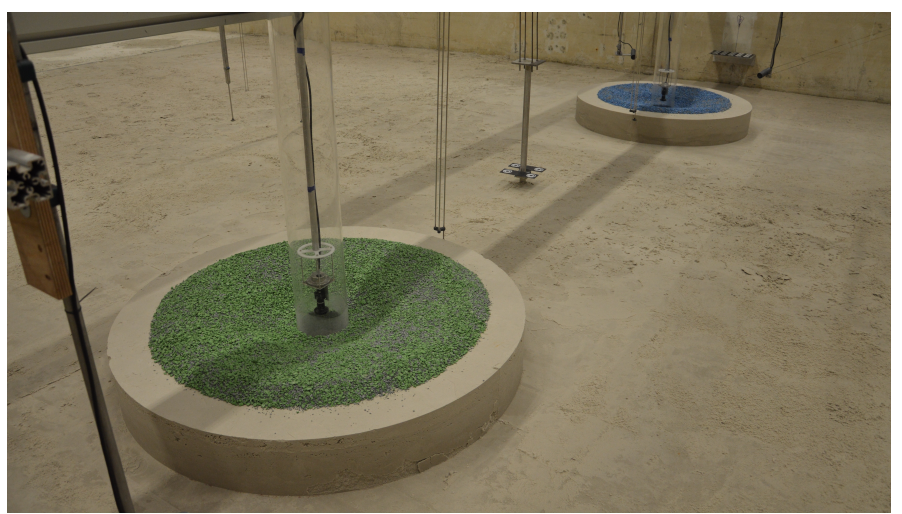

Figure 5. Overview of tests section before the start of test C. At both piles a 3-9" scour protection is installed.

\subsection{Simulation of morphologic change}

The scour protection concept involves rock material installed inside a pre-developed scour hole. To obtain a realistic scour hole shape for the physical model tests, the test programme started with an unprotected test investigating the scour hole shape in NOWF. A conservative scour hole was schematised based on the results of this first test series. In the following tests this schematised scour hole was excavated artificially, after which the scour protection was installed. The schematised shape of the scour hole can be seen in Figure 3. The model dimensions of the scour hole varied depending on the location within NOWF, as local variations in flow velocity and water depth would result in different scour holes.

The predicted large-scale seabed level lowering is modelled according to the methodology developed by Van Velzen (2012). Seabed level lowering is simulated by means of an artificial sill of sand, which is eroded under a series of tidal currents.

The followed construction sequence is: 1) placing a mould of the sill around the monopile, 2) filling the mould with sand and densely compacting it, 3) excavating the artificial scour hole from the sill and 4) installing the scour protection inside the scour hole on top of the sill. The resulting situation before the tests is visible in Figure 5, where sills of $6 \mathrm{~m}$ (to scale) are present. Before filling of the basin steep $45^{\circ}$ sandy support bunds were constructed against the sides of sill to ensure the stability of the sill when filling the basin. These bunds slowed down the initial erosion processes, but otherwise did not influence the falling apron behaviour of the scour protection.

\subsection{Test Programme}

The test programme is given in Table 1 . With the exception of the first test series ("O"), which only considered tidal conditions, each test series involved two types of tests: 1) tidal tests, in which several cycles of alternating ebb and flood current were simulated to launch the falling apron and edge-scour effects were investigated and 2) storm tests, which involved three consecutive storm conditions with increasing severity. A sea state of 6 hours is simulated, thus testing more than 2000 waves per test.

The hydrodynamic conditions varied depending on the monopile location investigated in each test series. Water depths are given in Table 1; the tested storm conditions can be found in Table 2 . The current velocity varied in each individual test, and was further adapted for cases with a morphological scenario. The current velocity was $0.17 \mathrm{~m} / \mathrm{s}$ in the storm with a return period of 1 year (hereafter RP1-storm) without morphological scenario and increased up to $0.36 \mathrm{~m} / \mathrm{s}$ in the most severe test. 
Table 1. Test programme.

\begin{tabular}{cccccc}
\hline $\begin{array}{c}\text { Test } \\
\text { Series }\end{array}$ & $\begin{array}{c}\text { Depth, } \\
\mathbf{h}_{\text {w;tal }}\end{array}$ & Pile & $\begin{array}{c}\text { Protection } \\
\text { Layout }\end{array}$ & $\begin{array}{c}\text { Morphologic } \\
\text { scenario }\end{array}$ & $\begin{array}{c}\mathbf{h}_{\text {MOR }} / \\
\mathbf{h}_{\text {w;tal }}\end{array}$ \\
\hline \multirow{2}{*}{ O } & \multirow{2}{*}{$0.422 \mathrm{~m}$} & 1 & - & Scour test & 1 \\
& & 2 & - & Scour test & 1 \\
A & \multirow{2}{*}{$0.422 \mathrm{~m}$} & 1 & $1-\mathrm{ND}$ & Initial situation & 1 \\
& & 2 & $1-\mathrm{HD}$ & Initial situation & 1 \\
B & \multirow{2}{*}{$0.531 \mathrm{~m}$} & 1 & $1-\mathrm{ND}$ & Winnowing* & 1 \\
& & 2 & $1-\mathrm{ND}$ & $0.109 \mathrm{~m}$ lowering & 0.21 \\
C & \multirow{2}{*}{$0.535 \mathrm{~m}$} & 1 & $2-\mathrm{ND}$ & $0.218 \mathrm{~m}$ lowering & 0.41 \\
& & 2 & $1-\mathrm{ND}$ & $0.218 \mathrm{~m}$ lowering & 0.41 \\
D & \multirow{2}{*}{$0.553 \mathrm{~m}$} & 1 & $2-\mathrm{ND}$ & $0.291 \mathrm{~m}$ lowering & 0.53 \\
& & 2 & $3-\mathrm{ND}$ & $0.291 \mathrm{~m}$ lowering & 0.53 \\
E & \multirow{2}{*}{$0.738 \mathrm{~m}$} & 1 & $3-\mathrm{HD}$ & $0.364 \mathrm{~m}$ lowering & 0.49 \\
& & 2 & $3-\mathrm{ND}$ & $0.364 \mathrm{~m}$ lowering & 0.49 \\
F & \multirow{2}{*}{$0.393 \mathrm{~m}$} & 1 & $3-\mathrm{HD}$ & $0.291 \mathrm{~m}$ lowering & 0.74 \\
& & 2 & $3-\mathrm{HD}$ & $0.204 \mathrm{~m}$ lowering & 0.52 \\
\hline
\end{tabular}

*The indicative winnowing test is not part of this study.

Table 2. Hydrodynamic conditions in storm tests.

\begin{tabular}{cccc}
\hline Return period & $\mathbf{H}_{\mathbf{m} \mathbf{0}}$ & $\mathbf{T}_{\mathbf{p}}$ & Notes \\
\hline 1 year & $0.14 \mathrm{~m}$ & $2.16 \mathrm{~s}$ & Used in all test series \\
10 year & $0.17 \mathrm{~m}$ & $2.69 \mathrm{~s}$ & Not used in test series D \\
50 year & $0.18 \mathrm{~m}$ & $2.96 \mathrm{~s}$ & Used in all test series \\
100 year & $0.19 \mathrm{~m}$ & $3.07 \mathrm{~s}$ & Only used in test series D \\
\hline
\end{tabular}

\section{ANALYSIS OF TEST RESULTS}

In this chapter three topics regarding the test results are highlighted: the falling apron behaviour of the scour protection, the observed deformation patterns and the overall performance of the scour protection.

\subsection{Falling apron effect}

Falling apron behaviour for monopile scour protections has not been tested so extensively, or for such extreme seabed level variations. The tests show that the concept works well, even for a seabed level lowering of $10 \mathrm{~m}$. The falling apron behaviour was observed by means of underwater cameras aimed at both the upstream and downstream side of the scour protection. An example image of a launching fallingapron can be seen in Figure 6.

\subsubsection{Falling apron behaviour}

Analysis of the movies of the tidal tests showed that the falling apron launched relatively fast initially. Especially at the sides of the sandy sill erosion occurred at a high rate. As the first rocks reached the surrounding seabed (i.e. had travelled all the way down the slope), the launching process slowed down. Once stabilised the launched falling apron covered the slopes all around the scour protection, resulting in a submerged protection island (see Fig. 7). The slopes have an angle of approximately 30 degrees.

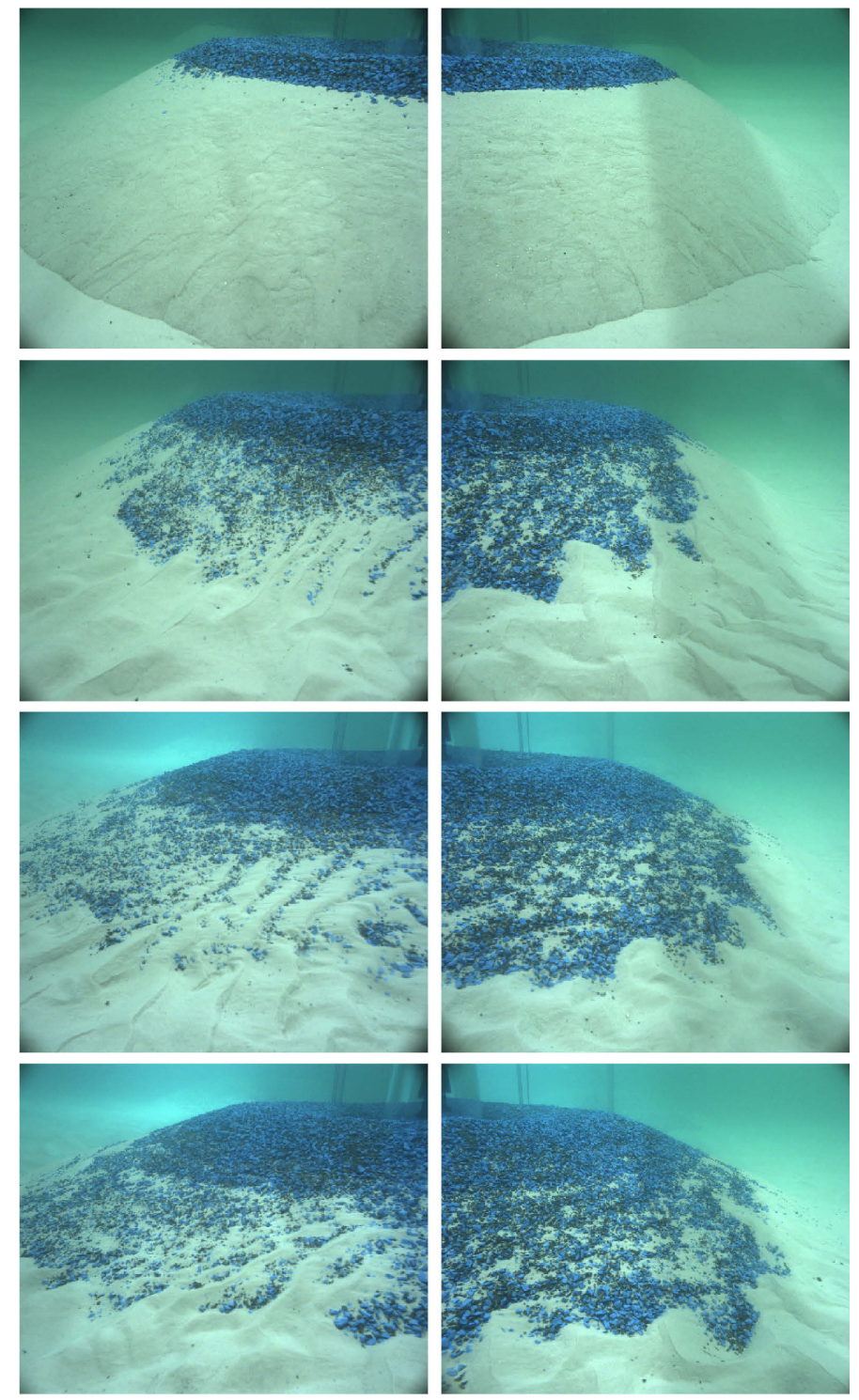

Figure 6. Underwater camera observations of several stages of launching the falling apron. Note that some sediment ripples have migrated half way up the slope, this is a scale effect.

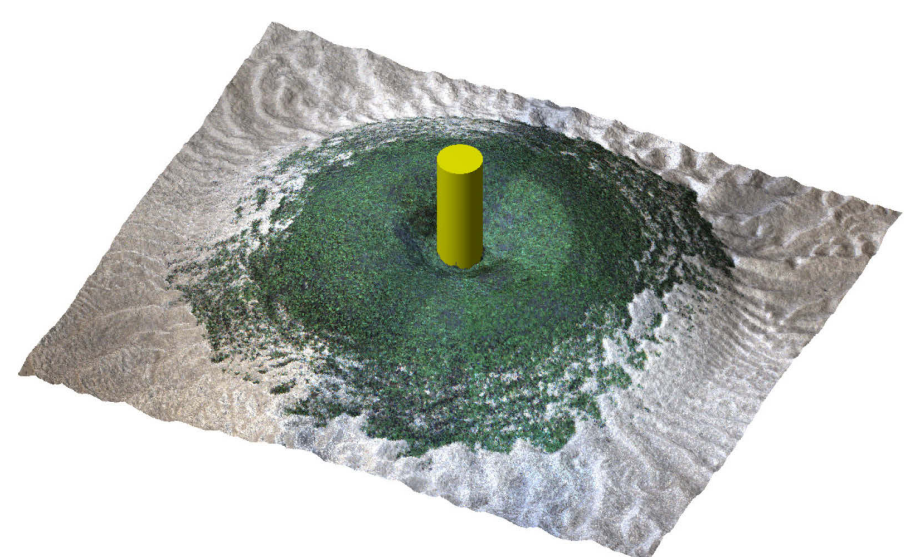

Figure 7. 3D colour image of pile 2 after launching the falling apron in test series D.

During the first wave test the falling apron typically resettled slightly and the profile became less steep. This is typically caused by material rolling down the slope from higher up. The process can also be seen very clearly in Figure 8 , which shows a cross-section of the bathymetry at the side of the pile during test series E. 


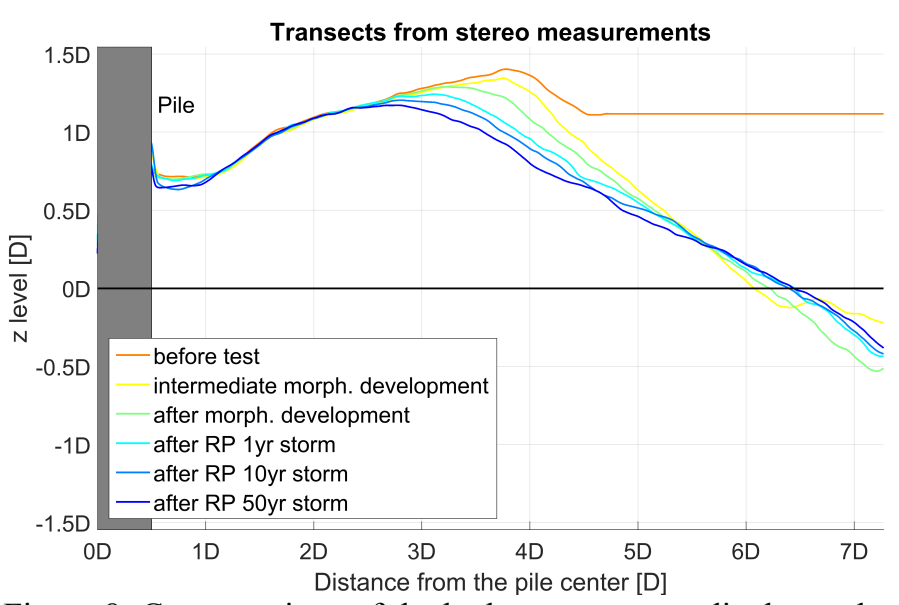

Figure 8 . Cross-sections of the bathymetry perpendicular to the tidal axis, during different phases of test series E.

The analysis of the underwater camera images further showed that no segregation of the material occurred. Both large and small particles in the rock grading showed the same falling apron behaviour and arrived at the bottom of the slope at the same time. This could also be determined by visual inspection of the launched falling apron: the larger particles in the grading (that were painted) and the smaller particles (unpainted) were distributed equally along the slopes.

\subsubsection{Differences due to rock density}

The effect of rock density on the falling apron behaviour was also investigated. In test series E, two identical scour protection layouts, but with different rock density, were tested under identical hydrodynamic conditions. In this test there was no observable difference in the falling apron behaviour of high density rock (at pile 1) and normal density rock (at pile 2). Also the end result (the fully launched falling apron) after the tidal test showed no significant difference. Under wave conditions the behaviour of the rock changed, because the high density rock is less mobile than normal density rock. This manifested itself mainly through more pronounced deformation patterns close to the pile and in the wake of the pile. At the slopes no deformation was observed and both protections had a resulting angle of approximately 30 degrees.

\subsection{Observed deformation patterns}

The stereophotography technique allowed for accurate measurement of the deformation of the scour protection. Some characteristic deformation patterns are described below in relation to the hydrodynamic condition.

\subsubsection{Deformation patterns in scour protections}

Typically three zones with downward deformation are observed in dynamically stable scour protections. A difference plot showing such deformations for a conventional scour protection on a flat seabed can be found in Figure 9. The incident wave and current direction is from the left.

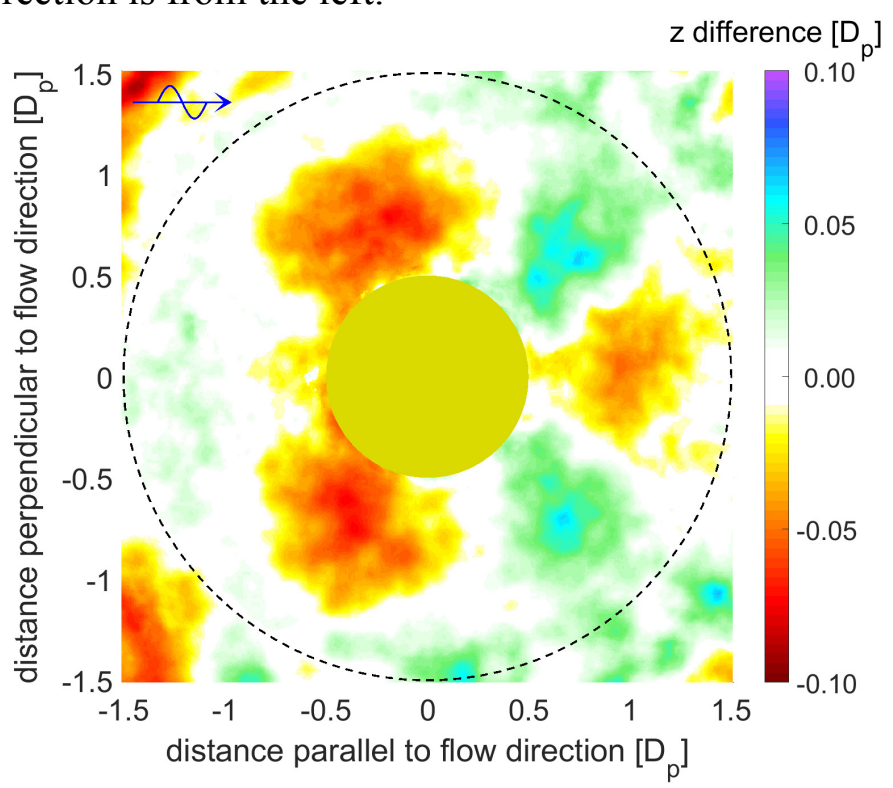

Figure 9. Typical deformation of a scour protection around a monopile. All dimensions normalised with the pile diameter. Incident wave direction is from the left.

Two 'lobes' can be discerned at approximately 60 degrees from the incident direction. The deformation that occurred here is caused by amplification of the bed shear stresses due to flow contraction around the structure (e.g. Sumer and Fredsøe (2001)). In conventional scour protections this deformation is observed under all conditions that are able to mobilise the protection material. It is independent of the $\mathrm{KC}$ regime, although the severity of the deformation varies.

Another zone with downward deformation is found in the wake of the pile. This zone is associated with lee-wake vortex shedding. In conventional scour protections this type of deformation is not always present, because lee-wake vortex shedding only occurs for higher $\mathrm{KC}$ regimes $(\mathrm{KC}>6$, Sumer and Fredsøe (2002)).

Furthermore two lobes with upward deformation can be observed at approximately 135 degrees from the incident wave direction. Part of the material that was removed from the downward deformation zones is transported towards these two locations.

\subsubsection{Observed deformation for NOWF}

In the milder storm conditions of the test programme the two deformation lobes at the upstream side of the pile were absent. This indicates that the sheltered position of the rock inside the scour hole (at a level of $-2 \mathrm{~m}$ relative to the original seabed) indeed reduces the hydrodynamic loading experienced by the rock. In more severe hydrodynamic conditions the deformation did appear.

The wake-deformation was observed in all test series where the scour protection was located higher in the water column. The two lobes with upward deformation were observed in all test series as well; 
even when the deformation due to bed shear stress amplification was absent (i.e. the two upstream zones with downward deformation). This means that the rock displaced due to lee-wake vortex shedding is moved in upstream direction down the slope, towards the more sheltered location close to the pile.

In test series E the rock close to the pile was painted blue, while the rock further away was painted green. This was done to investigate which material is transported towards the two upward deformation zones. It showed that in the first (mild) test, indeed green rock moved closer to the pile, proving that at least part of the material in these two lobes comes from the lee-wake vortex deformation zone. In the subsequent tests in this series, the material was removed again and also blue rock (from close to the pile) was present in the wake-deformation zone behind the pile. This is illustrated in Figure 10, which shows a colour image of the bathymetry after the last test in series E. This image was obtained by means of stereophotography.

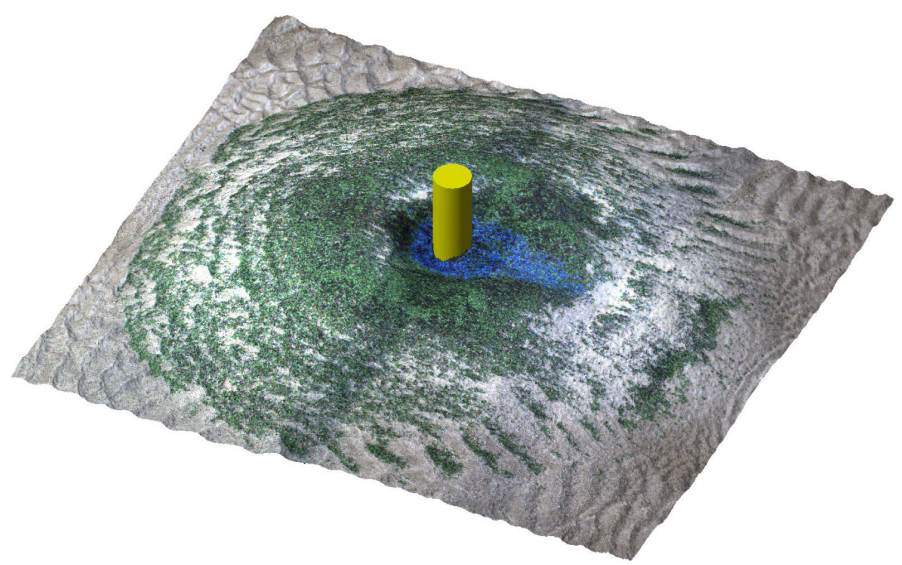

Figure 10. 3D colour image of pile 2 after test series E.

\subsubsection{Deformation of the launched falling apron}

Close to the pile $(<1$ pile diameter from the pile face) the scour protection experienced limited deformation. The sheltered position inside the scour hole ensured that no unacceptable deformation occurred. Further away from the pile, where the material is located in the shallower parts of the scour hole more deformation was observed. While the amplification of the bed shear stresses reduces significantly further away from the pile (Sumer and Fredsøe, 2002), this was apparently offset by the increased hydrodynamic loading due to its position higher up in the water column.

The most severe deformation was however observed at the flanks of the submerged scour protection island. Deformation at the two sides perpendicular to the incoming wave direction in some cases caused progressive failure of the scour protection. The presence of the island causes flow contraction itself, resulting in locally higher bed shear stresses at the two flanks of the island. At the same time the scour protection is located on a slope, reducing the stability of the rocks. These two effects, combined with the relatively small layer thickness of the launched falling apron caused exposure of the underlying seabed at the flanks of the submerged island.

Once exposed, the sediment quickly eroded. When sufficient material was still available higher up on the slope, the scour protection would selfrepair: new falling apron material would roll down the slope, covering the exposed sediment and stopping the erosion. In some cases however this new material was then displaced as well, resulting in a new local scour hole, gradually reducing the extent of the island, until the material close the pile began to function as falling apron material as well. This type of flank-failure of the scour protection occurred especially when the scour protection was located relatively high in the water column (test D and F), and showed less correlation with the actual height of the falling apron.

\subsection{Performance of scour protection}

The performance of the scour protection in each test is classified based on the observations during the test and the bathymetry measurements before and after each test. The outcome of each test is defined based on three criteria:

1. Sufficient remaining base extent $\left(4 * \mathrm{D}_{\text {pile }}\right)$;

2. Sufficient remaining layer thickness $(0.8 \mathrm{~m})$;

3. Pile fixation level maintained.

The first criterion checks whether part of the base extent of the scour protection was used as falling apron material, or transported away under severe hydrodynamic action. The second criterion is mainly related to the filter function of the scour protection. The third criterion specifically checks whether the fixation level at the pile base is still maintained. It is possible that scour protection fails close to the pile, resulting in local scour, which is subsequently stopped by material rolling from further away into the newly developed scour hole. In that case the original pile fixation level is not maintained, while the layer thickness criterion is not exceeded.

Based on these three criteria, four different classifications were defined:

1. No significant damage. Minor deformation, the overall shape of the scour protection is maintained and all criteria are still fulfilled.

2. Increased monitoring recommended. Deformation observed, but no immediate risk is identified. Increased monitoring is recommended, as an additional extreme event could cause more severe deformation or even failure.

3. Maintenance required. The scour protection is at risk of failure. Immediate repair works are required to mitigate the situation and prevent progression towards failure of the scour protection. 
4. Failure of scour protection. The pile fixation level has been breached close to the pile, or insufficient rock is available to withstand further scouring, even under a normal tide.

The situation after each individual storm test was analysed and the outcome subsequently classified. This resulted in a total of 33 data points (6 test series times 3 storms times 2 piles, minus the indicative winnowing tests at 1 pile). From the classification two very obvious conclusions could be drawn: 1) layouts with a larger rock volume perform better and 2) layouts with high density rock are more stable than the same layout with normal density rock. In the following section the results are quantified.

\section{RELATION BETWEEN DEFORMATION AND HYDRODYNAMIC LOAD}

Only a weak correlation was found between the outcome of the test series for NOWF and the relative mobility of the rock. However, a good correlation was found between the stability of the scour protection material and a modified relative mobility parameter.

\subsection{Relative mobility as indicator of rock stability}

The relative mobility (MOB) of the rock is defined as the ratio between the bed shear stress on the rock particles exerted by the hydrodynamic load (combined current and waves) and the critical bed shear stress. Typically, when the undisturbed mobility (without the presence of a structure) is larger than 1 movement of rock can be expected. For scour protections one must also consider the amplification of the hydrodynamic load due to the presence of the structure. Deformation of a scour protection around a monopile is typically expected when the undisturbed mobility becomes larger than 0.4-0.5. This makes the relative mobility a valuable predictor of expected deformation of a scour protection (e.g. Raaijmakers et al. (2010) and Den Boon et al. (2004))

\subsubsection{Calculation of the relative mobility}

The relative mobility of the rock gradings is quite sensitive to the adopted formulation to calculate the wave friction coefficient. Two commonly applied formulations are by Soulsby (1997) and Dixen (2008). The Soulsby formulation was developed for gravel and sand, whereas the Dixen approach, in addition to being more recent, has been explicitly extended for larger rock sizes. As a rule of thumb, the Soulsby formulation can be used when the wave roughness factor $\left(f_{w}\right)$ is higher than 10 and the Dixen formulation when this factor is lower than 10 . The equation to calculate $f_{w}$ varies for the two methods, but both are based on the wave orbital motion at the bed $\left(\mathrm{A}_{\mathrm{w}, \mathrm{a}}\right)$ and the equivalent roughness height $\left(\mathrm{k}_{\mathrm{s}}\right)$. In this paper the equivalent roughness height is taken equal to twice the value of $\mathrm{D}_{50}$. The wave orbital motion comes from the hydrodynamic conditions in each test. The resulting wave roughness factor $\left(f_{w}\right)$ was slightly larger than 10 for the mildest storm conditions, and became higher for the more severe conditions. Therefore only the formulation by Soulsby is used, which, compared to the approach by Dixen, results in slightly larger relative mobility values.

\subsubsection{Derivation of the modified relative mobility}

The outcome of the tests showed a weak correlation with the relative mobility of the rock. This led to development of a modified relative mobility, which takes into account the relative position of the material in the water column.

The wave orbital velocity is important for determining both the wave-related bed shear stress and the wave friction factor in a rough turbulent regime (Soulsby, 1997). When calculating the mobility one takes the wave orbital motion at seabed to arrive at the rock mobility at seabed. For the modified relative mobility calculation the orbital velocity is calculated at the level of the sill (i.e. the initial seabed level; $\mathrm{h}_{\mathrm{w} \text {;ini }}$ ), while the wave properties such as wavelength, height and period are determined by the surrounding deeper water depth after large-scale morphological development $\left(\mathrm{h}_{\mathrm{w} ; \max }\right)$. The underlying assumption is that the dimensions of the islands are relatively small compared to the wavelength; the waves will therefore not have time to adjust to the smaller water depth on top of the submerged island. This is illustrated in Figure 11. The approach resulted in a good correlation between modified relative mobility and the observed deformation of the scour protections.

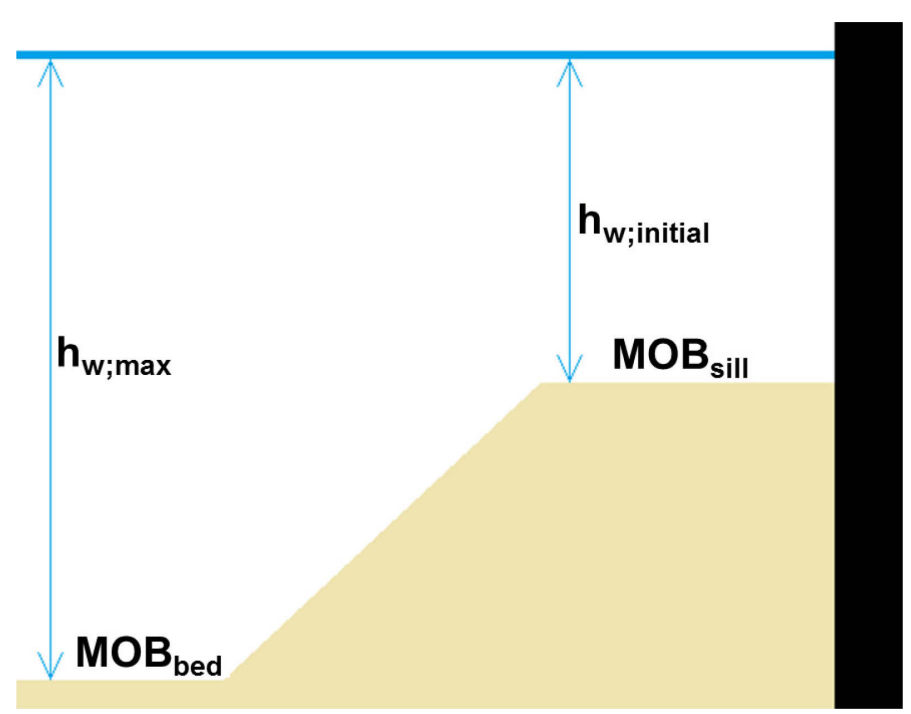

Figure 11. Schematisation of the protection island, with the water depth on top of the sill $\left(\mathrm{h}_{\mathrm{w} ; \text { initial }}\right)$, full water depth $\left(\mathrm{h}_{\mathrm{w} ; \max }=\right.$ $\mathrm{h}_{\mathrm{w} \text {;initial }}+\mathrm{h}_{\mathrm{w} \text {;MOR }}$ ) and the locations where the (modified) relative mobility is calculated, $\mathrm{MOB}_{\text {bed }}$ and $\mathrm{MOB}_{\text {sill. }}$. 


\subsection{Relation between performance of scour protection and modified relative mobility}

The use of the modified relative mobility at the top of the island $\left(\mathrm{MOB}_{\text {sill }}\right)$ combines the hydrodynamic loads (e.g. wave climate, current velocity) and rock parameters (e.g. $\mathrm{D}_{50}$, rock density) with the effect of the position of the scour protection in the water column. It thus allows for a straightforward comparison of all tested storm conditions and morphological scenarios.

Figure 12 graphs the simulated sill height and the calculated modified relative mobility for each test. The correlation is a bit occluded due to the different layouts used in the tests (indicated by the different colours of each marker). The increased volume of the larger layouts adds resilience to withstand the hydrodynamic loading that the scour protection is subjected to. This can also be observed in the test results. When looking at the individual layouts, a very clear correlation between test outcome (discussed in Section 4.3) and modified relative mobility is found.

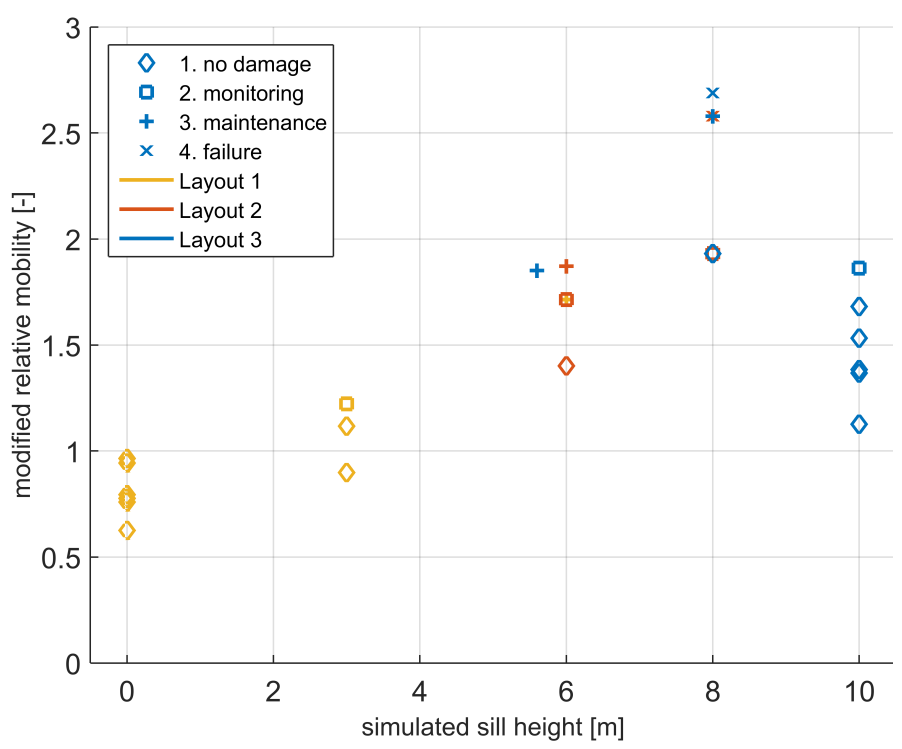

Figure 12. Simulated seabed level lowering versus modified relative mobility. The markers indicate the outcome of the test, the colour of the markers indicates the tested layout.

\subsubsection{Practical application}

Based on a detailed evaluation of the tested conditions a performance-bandwidth was determined for each scour protection layout: given the modified relative mobility the performance of each scour protection layout can be predicted. This is not only used for predicting the performance of the scour protection under the design conditions; the approach also allows for predictions of intermediate conditions (e.g. milder storm conditions and/or not fully developed morphological scenarios). This is extremely useful when planning monitoring strategies, predicting future maintenance costs and allows for optimising the scour protection layout at each individual pile location.
An example is given in Figure 13. This figure graphs the modified relative mobility of a (fictitious) rock grading for different seabed level drops, given a certain initial water depth $\left(\mathrm{h}_{\mathrm{w} \text {;ini }}\right)$. In this example the modified relative mobility has been calculated for two different storm conditions and two different rock densities. The patches provide an indication of the outcome should a certain modified relative mobility be exceeded: green indicates no significant deformation is expected; orange that maintenance will be required and red indicates expected failure of the scour protection.

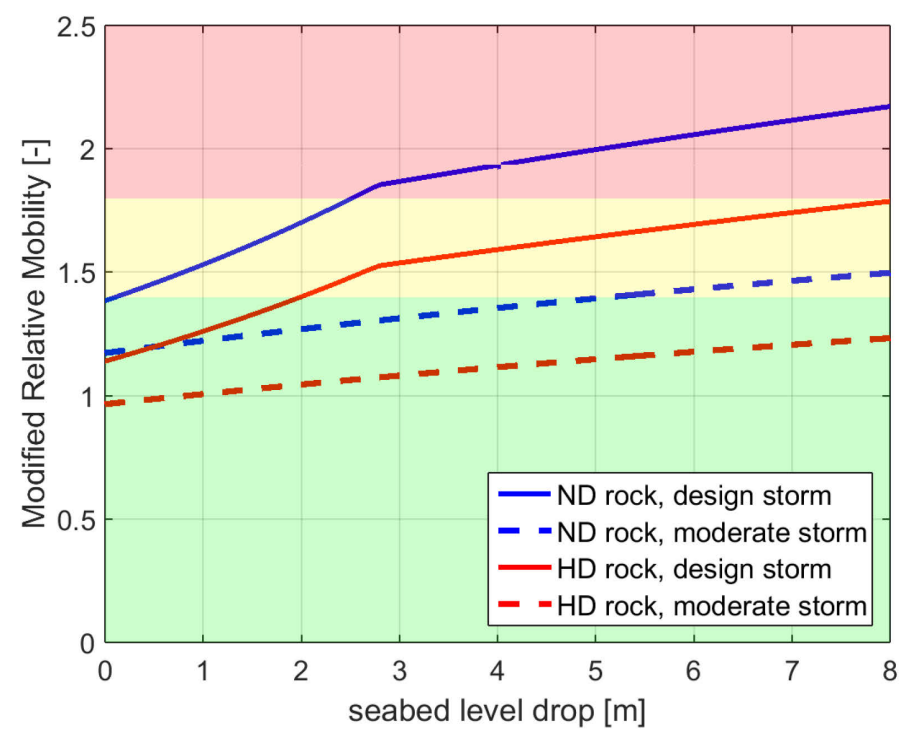

Figure 13. Example calculation of the relation between modified relative mobility and large-scale seabed level drop. The lines provide the relation for different rock density or hydrodynamic load, the patches provide an indication of the outcome should a certain modified relative mobility be exceeded.

\section{CONCLUSIONS \& DISCUSSION}

For Nordergründe offshore wind farm (NOWF), located in the German Bight, a single-graded scour protection, installed inside a pre-developed scour hole, is used to mitigate further scour development. The scour protection is designed to be flexible enough to handle both the design storm conditions and the significant morphological changes predicted for the wind farm area. The scour protection, consisting of a 3-9" rock grading, was subsequently tested in an extensive physical modelling programme. The tests investigated the behaviour of the dynamically stable scour protection in a highly morphodynamic environment.

Through the tests the scour protection concept for NOWF was validated. The tests showed that a functioning falling apron can be designed for locations with extreme seabed level lowering. Scenarios with a seabed level drop of up to ten metres were tested. Once launched, the falling apron formed a stable slope with an angle of approximately 30 degrees. 
The rock density did not have an effect on the falling apron behaviour of the scour protection.

In most of the tested storm conditions no failure of the scour protection occurred, under wave action the rock reshaped into a dynamically stable profile. Three typical deformation zones were observed. Erosion occurred at two 'lobes' on the upstream side of the pile. This deformation is associated with flow amplification around the pile and the resulting horseshoe vortex. Due to the sheltered position of the scour protection inside the scour hole, this deformation was less pronounced than observed for more conventional scour protections. The third deformation zone was observed in the wake of the pile. This deformation is associated with lee-wake vortex shedding and occurred even under mild storm conditions. After the initial reshaping of the profile, the scour protection remained (dynamically) stable.

Local failure of the scour protection sometimes occurred at the slopes of the protection. The launched falling apron had a reduced thickness at these locations, and the position on the slope reduced the stability of the rock. This type of failure was counteracted by increasing the rock volume available for the falling apron.

The performance of the scour protection layouts and morphological scenarios was systematically classified based on several criteria. A correlation was found between the outcome of each test and the modified relative mobility of the rock in the tested conditions. This modified relative mobility is calculated based on the schematised amplitude of the orbital velocities at the original seabed (i.e. at the top of the submerged protection island). For this schematisation the dimensions of the wave (height, period and length) are determined for the full water depth after morphological seabed lowering, but the orbital velocity decay is schematised according to the initial water depth. This method simplifies the rather complex flow of combined current and waves over the islands, but shows a good correlation with the test outcome.

The results of physical modelling test programme, combined with the observed relation between modified relative mobility and performance of the scour protection, allowed for optimisation of the scour protection at each of the foundations in NOWF. Furthermore the knowledge is useful for planning the monitoring strategy and predicting future maintenance costs after extreme storm events.

\section{REFERENCES}

De Sonneville, B., Joustra, R. \& Verheij, H. Winnowing at circular piers under currents. Scour and Erosion: Proceedings of the 7th International Conference on Scour and Erosion, Perth, Australia, 2-4 December 2014, 2014. CRC Press, 203.

De Sonneville, B., Rudolph, D. \& Raaijmakers, T. C. 2010. Scour reduction by collars around offshore monopiles. International Conference on Scour and Erosion. San Francisco.

Den Boon, J., Sutherland, J., Whitehouse, R., Soulsby, R., Stam, C., Verhoeven, K., Høgedal, M. \& Hald, T. Scour behaviour and scour protection for monopile foundations of offshore wind turbines. Proceedings of the European Wind Energy Conference, 2004.

Dixen, M., Hatipoglu, F., Sumer, B. M. \& Fredsøe, J. 2008. Wave boundary layer over a stone-covered bed. Coastal Engineering, 55, 1-20.

Hoffmans, G. J. C. M. 2012. The influence of turbulence on soil erosion, Eburon Uitgeverij BV.

Raaijmakers, T., Liefhebber, F., Hofland, B. \& Meys, P. 2012. Mapping of 3D-bathymetries and structures using stereophotography through an air-water-interface. Coastlab, 12, 17-20.

Raaijmakers, T. \& Rudolph, D. Time-dependent scour development under combined current and waves conditionslaboratory experiments with online monitoring technique. Proc. 4th Int. Conf. Scour Erosion, ICSE, Tokyo, 2008. 152-161.

Raaijmakers, T., Van Oeveren, M., Rudolph, D., Leenders, V. \& Sinjou, W. Field performance of scour protection around offshore monopiles. Scour and Erosion, 2010. ASCE, 428339.

Soulsby, R. 1997. Dynamics of marine sands: a manual for practical applications, Thomas Telford.

Sumer, B. M. \& Fredsøe, J. 2001. Scour around pile in combined waves and current. Journal of Hydraulic Engineering, 127, 403-411.

Sumer, B. M. \& Fredsøe, J. 2002. The mechanics of scour in the marine environment, World Scientific.

Van Velzen, G. 2012. Flexible scour protection around cylindrical piles, Delft University of Technology. 